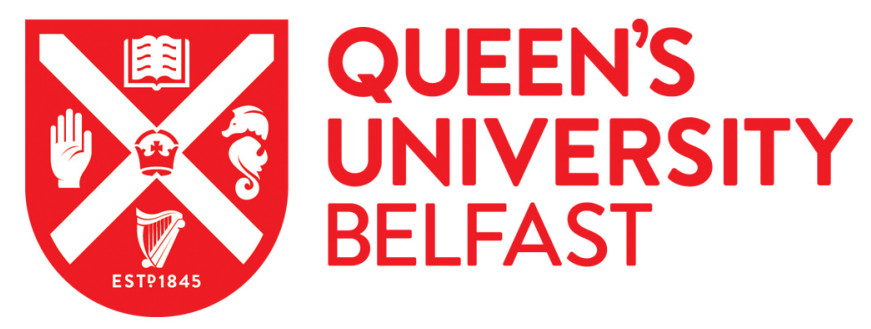

\title{
Highlights from the Faraday Discussion on lonic Liquids: From Fundamental Properties to Practical Applications, Cambridge, UK, September 2017
}

Aldous, L., Bendova, M., Gonzalez-Miquel, M., \& Swadzba-Kwasny, M. (2018). Highlights from the Faraday Discussion on Ionic Liquids: From Fundamental Properties to Practical Applications, Cambridge, UK, September 2017. 5261-5267. Paper presented at Faraday Discussion 154, Cambridge, United Kingdom.

https://doi.org/10.1039/C8CC90189A

Document Version:

Peer reviewed version

Queen's University Belfast - Research Portal:

Link to publication record in Queen's University Belfast Research Portal

Publisher rights

Copyright 2018 Royal Society of Chemistry. This work is made available online in accordance with the publisher's policies. Please refer to any applicable terms of use of the publisher.

\section{General rights}

Copyright for the publications made accessible via the Queen's University Belfast Research Portal is retained by the author(s) and / or other copyright owners and it is a condition of accessing these publications that users recognise and abide by the legal requirements associated with these rights.

Take down policy

The Research Portal is Queen's institutional repository that provides access to Queen's research output. Every effort has been made to ensure that content in the Research Portal does not infringe any person's rights, or applicable UK laws. If you discover content in the Research Portal that you believe breaches copyright or violates any law, please contact openaccess@qub.ac.uk. 


\section{Journal Name}

\section{COMMUNICATION}

\section{Highlights from the Faraday Discussion on lonic Liquids: From Fundamental Properties to Practical Applications, Cambridge, UK, September 2017}

Received 00th January 20xx, Accepted 00th January 20xx

DOI: $10.1039 / \times 0 \times x 00000 x$
Leigh Aldous, ${ }^{* a}$ Magdalena Bendova, ${ }^{* b}$ Maria Gonzalez Miquel ${ }^{* c}$ and Małgorzata SwadźbaKwaśny*d

www.rsc.org/

with the study; then, during the meeting, the authors delivered 5 minute presentations of their papers, followed by 30 minutes per paper discussion between the authors and the audience.

Prof Douglas McFarlane (Monash University, Australia) presented the introductory lecture entitled New dimensions in salt-molecular liquid mixtures: $A 4^{\text {th }}$ Evolution of Ionic Liquids (10.1039/C7FD00189D). Like many, Doug argued that the "salt melting below $100{ }^{\circ} \mathrm{C}$ " definition is obsolete, as the artificially set threshold temperature is limiting to the development of the field. Also, the "liquid comprised of ions" statement is problematic, since many IL systems have a measurable amount of non-ionic species, leading to the question about acceptable quantities of charge-neutral components. This semantic question, it was pointed out, was less important than the actual performance of ILs in the applications they were designed for. To support this, Doug provided an overview of successful applications of mixtures containing ionic and molecular liquids, in electrochemistry, bioactivity and tribology. It was suggested that all systems where Columbic interactions dominate the physicochemical behaviour should be considered within the realm of ILs - as opposed to dilute solutions (Figure 1). possible!

The chair of the Scientific Committee, Prof Christopher Hardacre (The University of Manchester, UK) opened with the introductory session, where he warmly welcomed the delegates attending. Afterwards, Chris Goodall and James Moore (Royal Society of Chemistry) introduced the unique format of the Faraday Discussions: pre-prints of the papers were sent to delegates in advance to allow to become familiar

\footnotetext{
a. Department of Chemistry, King's College London, London SE1 1DB, UK. Email: leigh.aldous@kcl.ac.uk

b. Address here.

c. The University of Manchester, School of Chemical Engineering and Analytical

Science, Manchester, M13 9PL, UK. Email:

maria.gonzalezmiquel@manchester.ac.uk

d. The QUILL Research Centre, School of Chemistry and Chemical Engineering, The

Queen's University of Belfast, Belfast, BT9 5AG, UK, Email:

m.swadzba.kwasny@qub.ac.uk
} 


\section{Journal Name}

\section{COMMUNICATION}

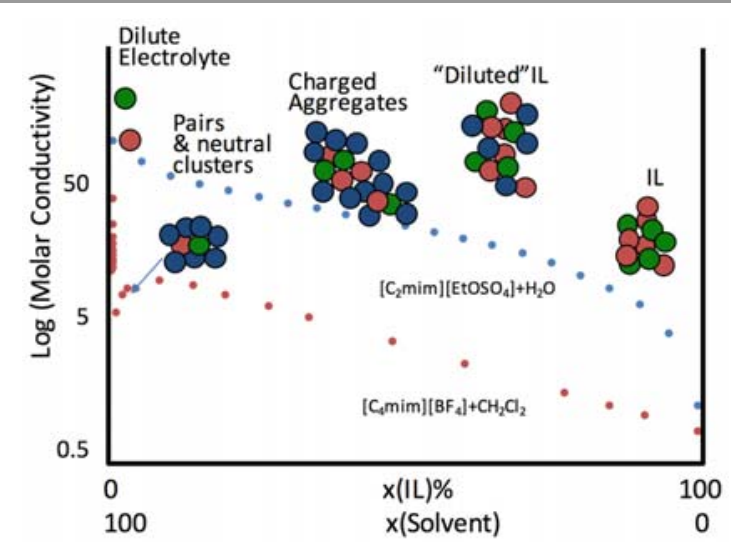

Figure 1. Molar conductivity trends in water $-\left[\mathrm{C}_{2} \mathrm{mim}\right]\left[\mathrm{EtOSO}_{3}\right]$ and dichloromethane - $\left[\mathrm{C}_{4} \mathrm{mim}\right]\left[\mathrm{BF}_{4}\right]$ mixtures. From 10.1039/C7FD00189D.

\section{Session 1: Phase Behaviour and Thermodynamics}

The regular meeting programme started with a session on phase behaviour and thermodynamics, in which fundamental contributions were presented and discussed. However, in keeping with the Discussion topic lonic liquids: from fundamental properties to practical applications, the talks and the ensuing discussions exemplified how such fundamental findings reach far into the field of applied chemistry.

The first half of Session 1 was chaired by Prof Margarida Costa-Gomes (CNRS and Université Clermont Auvergne, France), and focused on studies of the IL structure from the thermodynamic point of view. In liquid and solid phase, ILs have shown a wealth of structural and phase transition behaviour, from amorphous or nanostructured liquids, through cold crystallisation, to liquid crystals. To explore these phenomena in a systematic manner, Dr Tamar Greaves (RMIT University, Australia) prepared a series of protic ILs with nitrate and formate anions, and studied how the linear alkyl chain length on the cation influenced their structure and phase behaviour (10.1039/C7FD00148G). From a combination of differential scanning calorimetry, small- and wide-angle $\mathrm{X}$-ray scattering, and cross-polarised optical microscopy measurements, it appears that although the anion type influences the temperature of melting, ILs with a hexyl or longer alkyl substituent on the cation always show liquid crystalline phases that then melt into nanostructured liquids. This implied that the $\mathrm{H}$-bonding and ion-ion interactions have a greater impact on LC formation than the van der Waals interactions, which usually influence the crystal-to-liquid transition. In consequence, the organisation of the studied ILs in the amorphous and lamellar LC phase was elucidated.

Prof Hiroshi Abe (National Defence Academy, Japan) turned our attention to properties of ILs at extreme conditions (10.1039/C7FD00172J). Earlier studies on iodinebased ILs as electrolytes in dye-sensitized solar cells probed mass transfer of iodide ions. These paved the way for Hiroshi's work, in which phase behaviour of pure, stoichiometric, and non-stoichiometric ILs with iodide anions were studied at low temperatures and high pressures. Depending on the valence of the anion, crystalline or amorphous character of the ILs can be fine-tuned. However, non-stoichiometric ILs appeared to tend towards a more complicated behaviour at high pressures. These fascinating findings contribute to a better understanding of the polyiodide migration inside dye-sensitized solar cells that make use of ILs; an ultimate proof of the direct relationship between fundamental studies and crucial applications!

The first part of Session 1 was concluded by Prof Agilio Pádua (Université Clermont Auvergne, France), with a molecular dynamics study of the exfoliation of graphene and fluorographene in molecular and IL environment (10.1039/C7FD00169J). Though theoretical, the study may turn out to be essential in the design of single-layer 2D nanomaterials, such as graphene and fluorographene. The former being a conductor and the latter an insulator, inks of these suspended materials can for instance be used to print electronic devices on a variety of substrates, including textiles. Simulation was used to investigate how to "peel off" layers of graphene or fluorographene in different solvents. Indeed, the structure of the solvent appears to be a determining aspect in exfoliation, as the organisation of the interface between the graphene material and the solvent plays a crucial role. For instance, ILs with "flat" cations provide the best environment for the exfoliation process, as well as ILs with benzene or long alkyl side chain substituents. Apart from the clear influence of the surface tension, many 


\section{Journal Name}

\section{COMMUNICATION}

other properties that are still to be determined affect the exfoliation, motivating future studies. Nevertheless, a breakthrough in computational chemistry has already been achieved here, since different models had to be used to describe the exfoliation of a solid layer in a liquid.

The second part of Session 1, chaired by Dr Tristan Youngs (ISIS, UK), tackled more practical aspects of thermodynamics, related to material separation and process intensification. Ing. Carin Dietz (TU Eindhoven) addressed separation of hydroxymethylfurfural (HMF) and furfural (FF) after their synthesis from sugar-based biomass; separation was achieved by membranes based upon natural deepeutectic solvents (10.1039/C7FD00152E). HMF and FF are difficult to isolate due to their quick decomposition to lowvalue products under the synthesis conditions. The proposed in situ membrane separation thus appears to be a viable lowenergy alternative to distillation. The studied membranes provided good selectivity and efficient isolation of the two products, bearing the promise of a new, greener separation process.

Session 1 was closed by "the unforgettable" (according to the session chair) Prof Rob Atkin (University of Western Australia, Australia), who spoke about the use of ILs in pretreatment of algal biomass (10.1039/C7FD00158D). Microalgae are a sustainable and abundant source of biomass, but to obtain valuable products, lipids may have to be extracted first, in a pre-treatment step. According to some studies, ILs had the ability of breaking open the lipid barrier of algae cells, but their high cost and toxicity forebode their large-scale application. In this contribution, the use of less costly and environmentally friendly ILs based on natural substances was compared to conventional ILs, to assess the efficiency of this process. In the true spirit of fundamental research, it appears necessary to proceed case by case, to find the right balance between the energy efficiency of the process and sufficient yield of the product. After this, the first of two packed poster sessions went ahead, with discussion continuing into the night at the nearby Sir Issac Newton pub.

\section{Session 2: Structure and dynamics of ionic liquids}

The second session of the Faraday Discussions on Ionic Liquids was on the Structure and dynamics of ionic liquids, probed by the combination of computational and spectroscopic methods. The morning part of the session, chaired by Prof Chris Hardacre (The University of Manchester, UK) focussed on a range of X-ray techniques used in conjunction with modelling.

Dr Markus Mezger (Max Planck Institute for Polymer Research, Germany) talked about the molecular scale structure and dynamics at IL/electrode interface (10.1039/C7FD00171A), probed by the combination of in situ high energy $\mathrm{X}$-ray reflectivity (XRR) and impedance spectroscopy. The technique allowed the rearrangement of IL on the electrode surface to be monitored upon potential changes. Time-resolved experiments revealed three relaxation processes: a very fast (2 microseconds) ion transport in and out of the electrode surface, a slow by comparison (100 microseconds) molecular reorientation, and finally - a very slow lateral ordering within the first layer (min). Fast process was found to be transport dependent, implying diffusion, and the slow process was transportindependent, and therefore assigned to rotation. Slow kinetics of the rotational movement was justified by the interaction of ions with the electrode surface, which hindered the process.

Dr Millicent Firestone (Los Alamos National Laboratory, USA) presented on the self-assembly of ILs comprising the hydrophobic $\left[\mathrm{C}_{10} \mathrm{mim}\right]^{+}$cation and either $[\mathrm{SCN}]^{-},\left[\mathrm{NO}_{3}\right]^{-}$, or halide anions, upon their mixing with water (10.1039/C7FD00154A). The structuring of such mixtures was probed by small- and wide-angle X-ray scattering (SAXS and WAXS, respectively) and multiscale simulations. SAXS, which informs on the long-range interactions (low $Q$ ) was used to study the morphology of formed nanostructures. A complementary short-range (high $Q$ ) technique, WAXS, was used to study ion pairing and first shell coordination. Molecular dynamics simulations were carried out using inexpensive effective fragment potential (EFP) method, to model the ILs up to nanoscale. ILs with non-coordinating thiocyanide anions formed poorly-ordered lamellar structures irrespective of conditions; in contrast, ILs with coordinating halide anions allowed for huge tuneability of their nanostructuring. In conclusion, it was shown that molecular level organisation of the ionic region was found to propagate to the nanoscale level. 


\section{Journal Name}

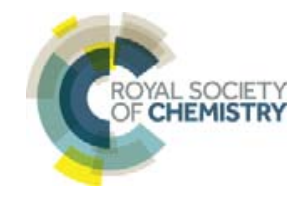

\section{COMMUNICATION}

Dr Patricia Hunt (Imperial College London, UK) delivered the third lecture of the morning, replacing of $\mathrm{Dr}$ Kevin Lovelock (University of Oxford, UK), who left the conference the night before. The birth of Kevin's baby, announced by Tricia at the beginning of the talk, is possibly the first childbirth reported during a Faraday Discussion conference talk. Scientific part of the talk (10.1039/C7FD00155J) was on the study of the atomic charge of sulfur in $\left[\mathrm{HSO}_{4}\right]^{-}$anion via $\mathrm{X}$-ray photoelectron spectroscopy (XPS), near edge X-ray absorption fine structure (NEXAFS) and Auger spectroscopy. The atomic charges have been calculated using three different approaches: charges from electrostatic potential using a grid based method (ChelpG), natural bond orbital (NBO) population analysis and Bader's atoms in molecules (AIM) approach. The two former methods were linearly correlating to the experimental data. In the discussion part, difficulties in modelling the large sulfur atom were highlighted.

In the final lecture of the morning session, Prof Jose $\mathbf{N}$. Canogia Lopes (University of Lisbon, Portugal) took us through the looking glass, into the world of charge-inverted ILs (10.1039/C7FD00139H). Taking a step-back approach and analysing the structure of commonly used cations and anions, it was noticed that, although there are hundreds of ILs with a bulky cation and an atomic anion (halide), only two examples of bulky anion with an atomic cation (alkali metal) could be found in the ILThermo database. Taking a very original approach, the authors set out to explore this 'broken symmetry' by developing and studying ILs combining potassium cations with 1-alkyl-3-methylcyclopentadienyl anions, $\mathrm{K}\left[\mathrm{C}_{n} \mathrm{C}_{1} \mathrm{Cp}\right](n=4,6)$, as 'mirror images' of common $\left[\mathrm{C}_{n} \mathrm{mim}\right] \mathrm{Cl}$ compounds - see Figure 2.
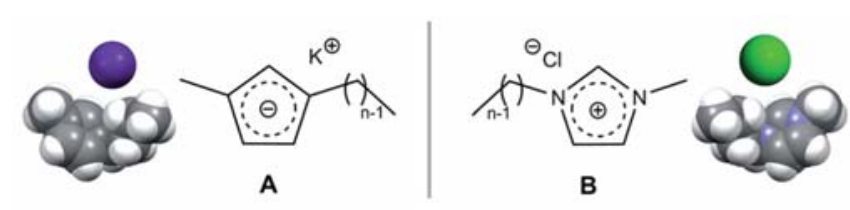

Figure 2. Potassium 1-alkyl-3-methylcyclopentadienyl (A) as the charge mirror image of 1-alkyl-3-methylimidazolium chloride (B), from 10.1039/C7FD00139H.

Despite close resemblances of the overall structure, significant differences in chemistry and physical properties were revealed between the 'analogues', originating from the presence of the aromatic ring on both components. Since the aromatic ring has negative charge, it interacted very differently with the $\mathrm{K}^{+}$cation, relative to more conventional ILs based upon the $\mathrm{Cl}^{-}$anion.

In the following discussion, the focus was on challenges in developing more advanced modelling tools. Both audience and the speakers shared some pitfalls they encountered, and discussed techniques for overcoming the need for very expensive simulations. In another strand of questions, ionic liquids were scrutinised through the looking glass: a range of other analogues of 'mirroring' ILs was proposed, such as phosphonium cations and borate anions. These were considered more convenient study models because, as the author remarked, ILs that spontaneously combust are not very popular.

Following the tea break, the second part of the session on structure and dynamics of ILs was chaired by $\mathrm{Dr}$ James Wishart (Brookhaven National Laboratory, US). Prof Barbara Kirchner (University of Bonn, Germany) opened this part with a lecture on structure and dynamics in ILs and their mixtures, probed by molecular dynamics simulations (10.1039/C7FD00166E). The focus was on the liquid structural features, such as hydrogen bonding, ion pairs and ion cages. When using polarisable force fields and increased temperatures, it was found that smaller coordination numbers, larger distances, and accelerated dynamics were achieved. In the discussion part, Barbara explained that in order to access 'cheaper' calculations of this type, one could use a smaller box, with ca. 100 ion pairs as opposed to 250 pairs used in this work; however, it was emphasised that long trajectories were absolutely required.

The second speaker, Dr John Holbrey (Queen's University Belfast, UK), discussed the structure and proton-transport pathways in protic imidazolium ILs with $\left[\mathrm{NTf}_{2}\right]^{-}$anion, studied by neutron scattering (DOI: 10.1039/C7FD00143F). In contrast to the authors' expectations (and most of the audience), no im $\cdots \mathrm{H} \cdots \mathrm{im}$ clusters were found directly from neutron scattering experiments. Although dimers were not directly observed, the centres of mass distances were of ideal length for proton hopping, pointing towards proton conductivity through flipping of the imidazolium ring: "we didn't spot the animal, but we see paths it makes in the forest". Since neutron scattering is a bulk structure 


\section{Journal Name}

\section{COMMUNICATION}

technique, not a dynamic (time-resolved) one, only an average structure can be seen; if there is $5 \%$ of proton transfer, it will not be observable.

Dr John Slattery (University of York, UK) closed the session with his lecture on probing the bulk structure of IL mixtures using SAXS (DOI: 10.1039/C7FD00167C). Rather than synthesising and studying numerous systems and relating their microstructure to properties, a time- and costefficient approach of mixing ILs to achieve a range of properties was used. Mixtures of $\left[\mathrm{C}_{2} \mathrm{mim}\right]\left[\mathrm{NTf}_{2}\right]$ and $\left[\mathrm{C}_{12} \mathrm{mim}\right]$ [NTf $f_{2}$ behaved like microemulsions, displaying non-ideal mixing behaviour.

The ensuing discussion spanned a range of interesting remarks. With regards to proton transfer, Prof Rob Atkin suggested that cluster analysis could provide significant structural insight for $\mathrm{H}$-bonding analysis. Prof Doug MacFarlane added that although $\mathrm{H}$-bond analysis is elusive, it is crucial for the $\mathrm{H}^{+}$transport mechanism and therefore important to study. On the bulk structure studies, Prof Margarida Costa Gomes emphasised that conductivity, viscosity and transport are not equilibrium properties, therefore cannot be discussed in terms of ideal mixing behaviour. $\mathrm{Dr}$ Wishart added that a similarly-looking macroscopic behaviour may be related to different phenomena on a molecular level.

\section{Session 3: Electrochemistry}

The third session covered Electrochemistry, with the short name encompassing a breadth of topics. The five papers up for discussion filled the afternoon of the second day, and the discussion was chaired by Drs Tristan Youngs (ISIS, UK) and Johan Jacquemin (Queen's University Belfast, UK).

The after-lunch discussion was opened by Dr Abhishek Lahiri (a Senior Scientist from Technische Universität Clausthal, Germany) and Prof Masayoshi Watanabe (Yokohama National University, Japan). Dr Lahiri's paper (10.1039/C7FD00121E) reported the anomalous electroless deposition of less noble metals in ILs; this was specifically noted on copper substrates. Detailed investigation involved electrochemistry, in situ XPS, mass spectrometry and in situ AFM. Changes in speciation in the IL environment were attributed to the observed effect, which was not predicted from the aqueous galvanic electrochemical series. The deposits obtained by electroless deposition from IL were demonstrated to be potential anodes for both Li-ion and $\mathrm{Na}$ ion batteries. In the resulting discussion, Dr Lahiri was grilled regarding how the mass spectrometry experiments were performed, the potential volatility of the solutes under the utilised conditions, and how this might have effected the speciation of the solutes; the discussion highlighted the very challenging nature, but necessary need, of determining how and why redox processes in IL systems can deviate significantly from those predicted by aqueous-based conventions.

Prof Watanabe reported results for novel protic ILs as $\mathrm{H}_{2}$ $\mathrm{O}_{2}$ fuel cells electrolytes, where a single water molecule was employed as amphoteric part of the IL (10.1039/C7FD00132K). This was achieved by combining water $\left(\mathrm{H}_{2} \mathrm{O}\right)$ with a super-acid or a super-base ( $\mathrm{H}$-Acid or Base) to form, in theory, $\left[\mathrm{H}_{3} \mathrm{O}\right][$ Acid] and [Base][OH]. Characterisation of thermal, physicochemical, and electrochemical properties of exemplar systems demonstrated that water and triflic acid formed a waterbased protic IL. Conversely, the super-base 1,8diazabicyclo[5.4.0]-7-undecene displayed limited proton transfer with water, resulting in poorer thermal and electrochemical properties than would have been expected of a protic IL. These observations were reflected by their performance in a fuel cell assembly. Discussion focussed upon the potential of these electrolytes in real-world devices, given the ubiquity of water and also its potential evaporation. For the triflic acid-based protic IL, having one water molecule per acid was optimum, with dilution by more water actually decreasing performance. However, the major limiting obstacle to long-term performance in conventional fuel cells was highlighted to be the highly corrosive nature of the super-acids and super-bases.

After the tea break, Prof Andrew P. Abbott (University of Leicester, UK) discussed the electrochemical and spectroscopic measurement of the inherent Brønsted acidity a range of deep eutectic solvents (DES) and ILs, and that of added organic acids (10.1039/C7FD00153C). Bromophenol blue was noted as a potential spectroscopic probe for such measurements. Glass-based electrodes were shown to display conventional (Nernstian) responses in diluted solutions of DES and ILs, and non-Nernstian responses in concentrated or pure DES and ILs. Nevertheless, the 


\section{Journal Name}

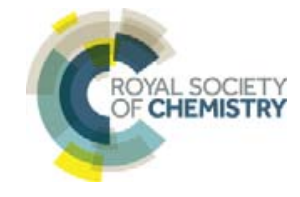

\section{COMMUNICATION}

responses mirrored that of the spectroscopic probe, highlighting the applicability of this method to investigate inherent acidity in DES and IL systems; the $\mathrm{pK}_{\mathrm{a}}$ of the investigated organic acids were also consistently ca. 0.5 units higher than that in aqueous media. However, of significant note was the fact that junction membranes can be $\mathrm{pH}$ dependent in DES and ILs-based systems, given the large difference in mobility between protons and the bulky cation, and therefore junction membranes require extra attention in such media.

Prof Laurence J. Hardwick (University of Liverpool, UK) continued the session with the oxygen reduction reaction in IL electrolytes (DOI: 10.1039/C7FD00170C); this electrochemical reaction was probed in detail at a gold electrode surface using Surface Enhanced Resonance Spectroscopy (SERS). This is of particular relevance to lithiumair batteries, where oxygen is reduced to the superoxide anion $\left(\mathrm{O}_{2}{ }^{-}\right)$, hence the effect of the IL cation was evaluated. Significant difference was observed between ILs based upon the relatively large 1-butyl-1-methylazepenium cation and the relatively small triethylsulfonium cation; the relatively poor and good electron accepting ability, respectively, influenced the Lewis basicity and thus behaviour of the electrogenerated $\mathrm{O}_{2}{ }^{--}$.

Finally, Prof Olivier Fontaine (Université de Montpellier, France) introduced the concept of biredox ILs (DOI: 10.1039/C7FD00174F). By having cations and anions each functionalised with a different redox species, this opens up additional redox chemistry that can be exploited for enhanced energy storage within supercapacitors. An imidazolium-based cation was functionalised with 2,2,6,6tetramethylpiperidinyl-1-oxyl (TEMPO), and a bis(trifluoromethylsulfonyl)imide-based anion was functionalised with anthraquinone; the resulting 'biredox IL' was then investigated dissolved in a conventional butylmethylimidazolium bis(trifluoromethyl-sulfonyl)imidebased IL. The introduced redox chemistry promoted additional pseudo-capacitance, on top of the significant capacitance inherent to ILs themselves, proving higher specific energy storage. Self-discharge within IL-based supercapacitor assemblies is an unwanted and limiting issue; however, the high charge and therefore low mobility of the 'charged' biredox IL was found to beneficially slow down the self-discharge process. Discussion highlighted that the chemistry of the biredox species was not entirely consistent with the expected chemistry of the separate moieties, but the complexity of the substituted species and high concentration (up to $0.5 \mathrm{M}$ ) were likely responsible.

Throughout, discussion was varied, but a recurrent theme was the need to generate a consistent series of reference potentials to fully compare and contrast IL systems with more conventional media. Also noted was that there were as many different reference electrodes as there were papers; a universal reference electrode (or at least a widely adopted one) would greatly facilitate this task.

\section{Conference Dinner and Loving Cup Ceremony}

After the second day bursting of interactive debates, it was time to continue sharing scientific views while enjoying social networking at the Faraday Discussion Conference Dinner. The banquet-style meal was served at The Dome Dining Hall of the Murray Edwards College, decorated with impressive artwork from the New Hall Art Collection, which includes creations from Turner Prize Winners. The pleasing evening concluded with the traditional Loving Cup Ceremony to toast "in piam memoriam of G.S. Marlow and Angela and Tony Fish". Mr Marlow was secretary and editor of the Society from 1928-1947, while Angela Fish worked for the RSC from 1968-1995 and pioneered organization of Faraday Discussion meetings, leaving a legacy along with her husband, which was used to establish the RSC Travel Grants scheme. In their memory, the so-called Faraday Loving Cup is used on the special occasion of the Conference Banquet to celebrate a traditional ceremony: the silver cup dating from 1728 filled with sweet red wine is passed around the assembled guests, starting from the table chaired by the President and the Organizing Committee, following an ancient 'bowing and sipping ritual' (Figure 3 ) that certainly makes another distinct feature of the Faraday Discussion event! 


\section{Journal Name}

\section{COMMUNICATION}

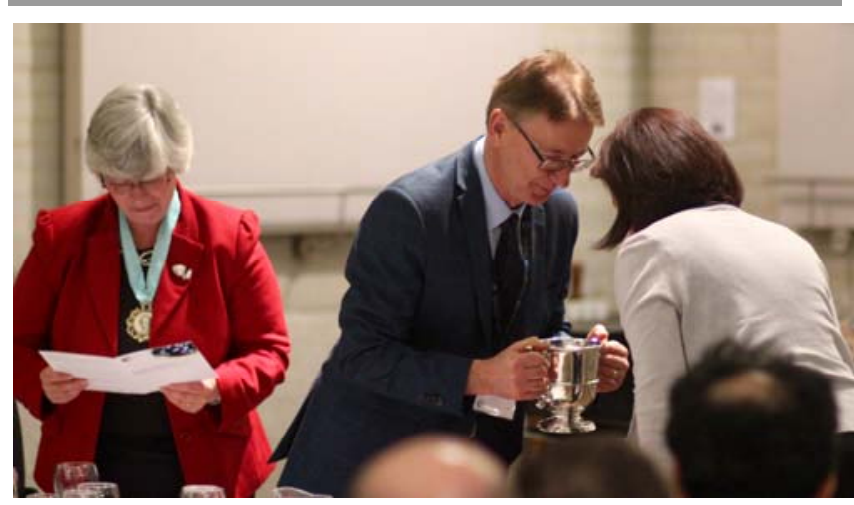

Figure 3. Conference Dinner and Loving Cup Ceremony.

\section{Session 4: Ionic Liquids at Interfaces}

The contributions presented during the fourth session of the Faraday Discussions aimed to provide a further understanding of the behaviour of ILs at different liquid, solid and gaseous interfaces. This is of fundamental interest to a broad range of practical applications, e.g. electrochemistry, nanotechnology, gas absorption, energy storage and catalysis. The first half of this morning session started with the presentation of Prof Susan Perkin (University of Oxford, UK), who focused upon recent insights into the interfacial structure and structural forces in mixtures of ILs and polar solvents (10.1039/C7FD00168A). This was achieved by combining both experimental studies and molecular simulations. The experiments centred around mica surface force balance (SFB) measurements in mixtures of ILs (i.e. [bmpyrr] $\left[\mathrm{NTf}_{2}\right]$ or [emim] $\left.\left[\mathrm{NTf}_{2}\right]\right)$, and propylene carbonate as polar molecular co-solvent. Experimental results were compared with molecular dynamic (MD) simulations, providing information of the molecular structure of the systems at a charged surface. A significant finding of this investigation involves a structural transition from chargeoscillatory to charge monotonic decay, leading to strong solvent layering in the systems when the ionic liquid is diluted with polar solvent.

The second work, presented by $\mathrm{Dr}$ Benjamin Cross (Université Grenoble Alpes, France), studied the nanomechanics and nano-rheology of ILs at dielectric and metallic interfaces (10.1039/C7FD00149E). A dynamic surface force apparatus (dSFA) was used to evaluate the nano-mechanical properties of the hydrophobic IL $[\mathrm{bmim}]\left[\mathrm{PF}_{6}\right]$ at Pyrex dielectric and metallic platinum solid surfaces. It was found that the thickness of the interfacial phase of the IL on the metallic surface was up to an order of magnitude larger than on the dielectric surface, although the elastic modulus of both interfacial layers were similar; moreover, hydrodynamic measurements supported that the thickness of both interfacial layers is not dependant on the distance between the surfaces, and that they do not exhibit a capillary-freezing mechanism.

The third contribution was presented by $\mathrm{Dr}$ Natalia Borisenko (Clausthal University of Technology, Germany), discussing how the presence of different precursors $\left(\mathrm{TaF}_{5}\right.$, $\mathrm{SiCl}_{4}, \mathrm{GaCl}_{3}$ ) affect the $\mathrm{Au}(111)$ deposition process and the solid/IL interfacial structure (10.1039/C7fD00165G). In particular, systems involving pyrrolidinium-based ILs paired with different anions, i.e. [TFSA] $]^{-}$[TfO $^{-}$and [FAP]', were evaluated by vibrational spectroscopy, to understand their structural changes upon addition of precursors. In situ Atomic Force Microscopy (AFM) also provided insights into the $\mathrm{Au}(111) / \mathrm{IL}$ interface. Overall, results indicate that the structure of the IL/electrode interface is different for each system, which ultimately determine the characteristics of the deposition process. It was found that $\mathrm{Ta}$ can be electrodeposited in [bmpyrr][TFSA]/Au(111) while Ga can be electrodeposited in [bmpyrr][TFSA] and [bmpyrr][TfO], with both the anion and the precursor having impact on the $\mathrm{Au}(111) / \mathrm{IL}$ electrical double layer structure, which affects the deposition process and the morphology of the deposit; moreover, increasing the concentration of the $\mathrm{SiCl}_{4}$ precursor alters the [bmpyrr][FAP]/Au(111) interface, leading to a double layer structure that ease the Si deposition process.

Afterwards, Dr Seiji Tsuzuki (National Institute of Advanced Industrial Science and Technology, Japan) requested to intervene to share with the audience his comments on the interaction of ionic species with surface conductors; he highlighted that interactions between ionic species and conductor are stronger than those of non-polar species due to the interaction with mirror charge, which play an important role in determining the distribution and orientation of ions near conductor surfaces; however, it was emphasised that attention should be paid when using simulation results of conductor surface since interaction with 


\section{Journal Name}

\section{COMMUNICATION}

mirror charge is usually not considered in most classical molecular dynamic (MD) approaches. During the discussions of the papers, the audience debated assumptions made on MD simulations for estimation of surface charge density; it was noted that the structural transition of IL solutions not only depends upon the concentration but also the surface charge. Highlights regarding the behaviour of ILs near charged surfaces were made, remarking that charge in the IL is not uniform and this should be reflected in the simulations; additionally, it was suggested to further evaluate the influence of the alkyl chain length in electrodeposition to comprehensively understand the behavioural trends as a function of the IL structure.

Following a morning tea break, the second half of the session resumed with the presentation of Prof Robert Jones (University of Nottingham, UK), regarding interaction and stabilisation of acetone, sulfur dioxide and water with ILs at low temperatures (10.1039/C7fD00146K). In particular, interactions between the small molecules and [omim] $\left[\mathrm{BF}_{4}\right]$ were evaluated through line of sight temperature programme desorption (LOSTPD), after being adsorbed (acetone) or co-adsorbed (i.e. sulfur dioxide and water) with the IL from the gas phase. It was found that the IL forms a highly porous structure upon vapour deposition, with the three small molecular species being stabilised by interaction with the ionic parts of the solvent, while destabilised states were related with adsorption onto the alkyl chains; additionally, in the case of water there appeared to be a range of stabilised states that could not be attributed solely to the porous structure, but to the ionic liquid's ability to modify the hydrogen bonding network of the solute.

Subsequently, Dr Simon Purcell (Heriot-Watt University, UK) presented his paper concerning composition determination of the vacuum-liquid interface in IL mixtures (10.1039/C7FD00175D). In this work, different imidazoliumbased IL mixtures composed of cations with different chain lengths $\left(C_{2}\right.$ vs. $\left.C_{12}\right)$, cations with different chain functional groups (alkyl vs. fluoro) and different anion mixtures ([BF $]^{-}$ vs. $\left[\mathrm{NTF}_{2}\right]^{-}$) were evaluated through reactive-atom scattering with laser-induced fluorescence detection (RAS-LIF), surface tension measurements and MD simulations; this enabled determining the surface composition of the IL mixtures and testing different theoretical models to estimate the composition of the systems from the surface tension of the pure components. Overall, results suggest that the component with lower surface tension dominates the surface of IL mixtures, following Henry's and Raoults's law-like behaviours at low and high concentrations respectively, with extended Langmuir models providing a sensible description of the observed trends.

Next, Prof Toshiyuki Itoh (Tottori University, Japan) presented his paper on the effects of ether oxygen atoms on the physical properties of piperidinium ILS $(10.1039 / C 7 F D 00142 H)$. This work reported the synthesis and provided a systematic evaluation of the physico-chemical properties of piperidinium-based ILs; it was highlighted that ether oxygen atoms on alkyl side chains allow further optimisation in their design for electrochemical applications. It was found that the position of the oxygen atom on the alkyl side chain has a significant impact upon the viscosity, ionic conductivity, and thermal and electrochemical stability of the piperidium ILs, with 1-methyl-1-(2propoxyethyl)piperidin-1-ium bis(trifluoromethylsulfonyl)imide ([PP $\left.1 \mathrm{PE}]\left[\mathrm{NTf}_{2}\right]\right)$ offering the best balance of properties for application as an electrolyte. Additional Density Functional Theory (DFT) calculations provided insights into the oxidation potentials of the ILs and predict their electrochemical window.

The last contribution was presented by Dr Octavian Pavel (University of Bucharest, Romania) regarding the behaviour of Solid Catalyst with an Ionic Liquid Layer (SCILL) in the oxidative $\mathrm{S}-\mathrm{S}$ coupling of thiols to disulfides (10.1039/C7FD00159B). This work reported preparation of heterogeneous catalyst coated by $\left[\mathrm{NTf}_{2}\right]$-based ILs paired with imidazolium and pyrrolidinium cations, which were deposited onto a variety of active solids (i.e. $\mathrm{Pt} / \mathrm{SiO}_{2}, \mathrm{Ru} / \mathrm{SiO}_{2}$, $\mathrm{Ru} / \mathrm{C}$ and Pt/OMS-2 at 5\% wt.). Afterwards, the application of the SCILLs in the aerobic oxidative coupling of aliphatic (1butanethiol) and aromatic (thiophenol) thiols to disulfides under mild conditions was explored. SCILLs provided high selectivities to disulphide, with the presence of the IL increasing the stability of the catalyst and allowing the recyclability of the solid material, due to their ability to reduce the adsorption strength of the sulphur species on the metal. 


\section{Journal Name}

\section{COMMUNICATION}

During discussion of the papers, several key points were highlighted, including the need for further evaluation of the IL pore structure and subsequent stabilisation/destabilisation of small molecules upon deposition if IL with shorter alkyl chains were used; the effect on leaching and osmotic pressure of the catalyst when transitioning from batch to flow processes, as well as the presence of water or other molecular solvents into the catalyst performance; and the need to evaluate the impact of ether-functionalised ILs on Li transport properties and their compatibility with Li batteries for energy storage. Overall, this session provided a deeper understanding of the interfacial behaviour, structure and interactions of IL systems, which is crucial towards developing target practical applications.

\section{Concluding remarks lecture and closing session}

After three days of relevant scientific contributions, stimulating debates and networking social events, the closing session of the Faraday Discussion was chaired by Prof Christopher Hardacre (The University of Manchester, UK). The concluding remarks lecture was presented by Prof Philip Jessop (Queen's University, Canada), who provided a review of the conference, discussed about emerging classes of ILs, and shared a vision for the future in his illustrative talk precisely entitled Where are we going? A key take-home message from his inspiring lecture is the urgent need to identify truly green ionic liquids, for which comprehensive Life Cycle Assessment (LCA) tools are required. It was emphasised that a green solvent should make a product or process have the least environmental impact over its entire life cycle. Therefore, the new generation of ILs (regardless if they are conventional or not, in pure state or as a mixture) should achieve a specific task more efficiently than any other liquid, while also representing the solvent causing the least impact (not only as a solvent but also in the specific process), more so than any other liquid would have done. 